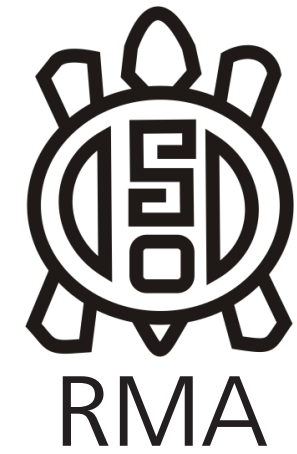

Arqueología

\title{
Un diente de tiburón fósil usado como objeto adorno-colgante en la localidad Arqueológica Punta Medanosa (costa norte de Santa Cruz)
}

\author{
A fossil shark tooth used as a pendant at the Punta Medanosa \\ archaeological locality (north coast of Santa Cruz) \\ Miguel Ángel Zubimendi*; Alicia Castro**, Pablo Ambrústolo*** \\ y Carolina Contreras ${ }^{* * * *}$
}

\begin{abstract}
*Miguel Ángel Zubimendi. CONICET. Unidad Académica Caleta Olivia, Universidad Nacional de la Patagonia Austral; División Arqueología. Museo de La Plata. UNLP. Paseo del Bosque s/n. La Plata, Argentina. C. P. 1900. Argentina. mikelzubimendi@gmail.com **Alicia Castro. División Arqueología. Museo de La Plata. UNLP. Paseo del Bosque s/n. La Plata, Argentina. C. P. 1900. Argentina. aliciacastro52@gmail.com ***Pablo Ambrústolo. CONICET. División Arqueología. Museo de La Plata. UNLP. Paseo del Bosque s/n. La Plata, Argentina. C. P. 1900. Argentina. pambrustolo@hotmail.com ${ }^{* * * *}$ Carolina Contreras. División Arqueología. Museo de La Plata. UNLP. Paseo del Bosque s/n. La Plata, Argentina. C. P. 1900. Argentina. carolinacontrerasromero@gmail.com
\end{abstract}

\begin{abstract}
Resumen
Durante los trabajos de muestreo de materiales arqueológicos en superficie en un conchero de la localidad Punta Medanosa se recuperó un diente fósil de tiburón, claramente asociado contextualmente a dichos restos. La ubicación del conchero sobre una matriz de dunas en erosión, asentado sobre cordones litorales del Holoceno tardío, permitieron afirmar que el hallazgo correspondía a un ecofacto, es decir, un producto natural que fue transportado desde donde afloran los fósiles hasta el sitio. Mediante trabajos de laboratorio, a nivel macro y microscópico, se constató que el ejemplar correspondía a un artefacto modificado mediante técnicas de pulido, probablemente en el marco de la confección de un objeto colgante para uso como adorno corporal. En esta nota se presenta una descripción de la pieza recuperada, así como los estudios que sustentan la identificación de las modificaciones antrópicas que confirman su caracterización como objeto adorno-colgante.
\end{abstract}

Palabras clave: "Isurus" hastalis; ecofacto; conchero; objeto de adorno personal

\begin{abstract}
During a surface sampling of archaeological materials on a shellmidden in the Punta Medanosa locality, a fossil shark tooth clearly associated was recovered. The location of the shellmidden on a matrix of erosion dunes, seated on late Holocene cordons, allowed us to affirm that this finding corresponded to a ecofact or biofact, that is, a natural product transported to this site. Subsequently, by means of macro and microscope laboratory analysis work, it was found that it was a modified artefact by polishing and notching, which would have allowed its use as a pendant, probably as a corporal adornment. In this note, a description of the recovered piece is presented, as well as the studies carried out to support its allocation as a corporal adornment.
\end{abstract}

Keywords: "Isurus" hastalis; ecofact; shellmidden; personal adornment

El diente fósil fue recuperado durante trabajos de recolección de artefactos líticos en superficie en el conchero La Señal, en la localidad arqueológica Punta Medanosa (Figura 1.a y b). La duna donde se emplaza el sitio se encuentra asentada sobre sobre cordones litorales compuestos por conchilla formados en momentos posteriores a 1500 años AP (Constante 2001, Medina et al. 2014). En el sitio se realizó un sondeo estratigráfico de $25 \times 25 \mathrm{~cm}$ de lado sobre un perfil expuesto de una lente densa de valvas de moluscos, la cual se hallaba inserta dentro de una duna de dimensiones medianas (Figura 1.c). En los perfiles del sitio y los materiales caídos producto de su erosión, se observaron gran cantidad de artefactos líticos y restos óseos de pinnípedos. Por encima de la lente de valvas se identificó una fina cubierta de sedimentos eólicos mezclados con restos arqueológicos. En la excavación se comprobó que se trataba de una única lente muy densa de restos malacológicos de aproximadamente 
$20 \mathrm{~cm}$ de potencia. Principalmente se registraron valvas de Mytilus edulis, y en menor medida Aulacomya atra y Nacella magellanica, aunque también se identificaron Perumytilus purpuratus, Siphonaria lessoni, Crepipatella sp. y Adelomelon sp., entre otras especies de moluscos. A nivel estratigráfico, se destaca la presencia de restos óseos de pinnípedos en altas frecuencias. La densidad de artefactos líticos es baja, entre dichos restos predominan los productos de talla. En el sector inferior de la lente de valvas se observó una camada de huesos de pinnípedos compuesta por varios ejemplares que se hallaban juntos y superpuestos entre sí. La datación radiocarbónica de carbones dispersos ubicados en la porción basal del sondeo arrojó un fechado de $2160 \pm 80$ años AP (LP3475). A nivel superficial, se realizaron dos cuadrículas de recolección de $2 \times 2 \mathrm{~m}$, sobre la lente donde se realizó el sondeo. En las mismas se registraron 31 artefactos líticos, en su mayoría se trata de productos de talla (lascas angulares y de arista de tamaño pequeño y mediano-pequeño confeccionadas sobre sílex, calcedonia traslúcida y obsidiana) y un núcleo de xilópalo. En una de las mencionadas cuadrículas se identificó el diente fósil de tiburón analizado en el presente trabajo.

\section{El diente fósil de tiburón}

La pieza fósil recuperada corresponde a un diente lateral, con la corona notablemente curvada (Figura 2). Se trata de un ejemplar con bordes cortantes lisos y suaves. La corona es aplanada labio-lingualmente con la base relativamente ancha. La cara labial es plana y la cara lingual ligeramente convexa. Ambos filos están bien preservados, con el borde mesial de contorno convexo y el distal notoriamente cóncavo en la base de la corona y recto hacia la región apical (Figura 2). No presenta cúspides laterales, la raíz está dañada y, en mayor medida, ausente. El ejemplar presenta un largo máximo de $32,03 \mathrm{~mm}$ y un ancho máximo de 25,14; el espesor de la corona es de 4,67 $\mathrm{mm}$ y el de la parte conservada de la raíz es de 7,68 $\mathrm{mm}$.

Sobre la base de los caracteres antes mencionados se puede afirmar que la morfología presente en este diente es totalmente coincidente con la descripta para la especie "Isurus" hastalis. Este tiburón es una forma extinta de la familia Lamnidae, cercanamente emparentada con el actual tiburón blanco (Carcharodon carcharias). Los registros de "Isurus" hastalis en Argentina son muy abundantes en casi todos los niveles marinos Terciarios (Arratia y Cione 1996) y son especialmente comunes en los sedimentos referenciados al mar Patagoniense (Mioceno inferior) de Chubut y Santa Cruz (Cione 1988). La asignación sistemática de este taxón a nivel de género está en discusión, por lo que se consigna entre comillas. Al respecto, existen diversas posturas (por ejemplo, Cione et al. 2012; Ehret et al. 2012; Ward y Bonavia 2001, entre otros). Estos tipos de dientes de tiburón han sido documentados en los afloramientos de la formación Monte León del Mioceno temprano (Cione 1988), la cual tiene una amplia distribución en el noreste de Santa Cruz, y en particular en las cercanías de Punta Medanosa, hacia el oeste (Panza et al. 1994). Las distancias mínimas estimadas en línea recta del sitio La Señal respecto de

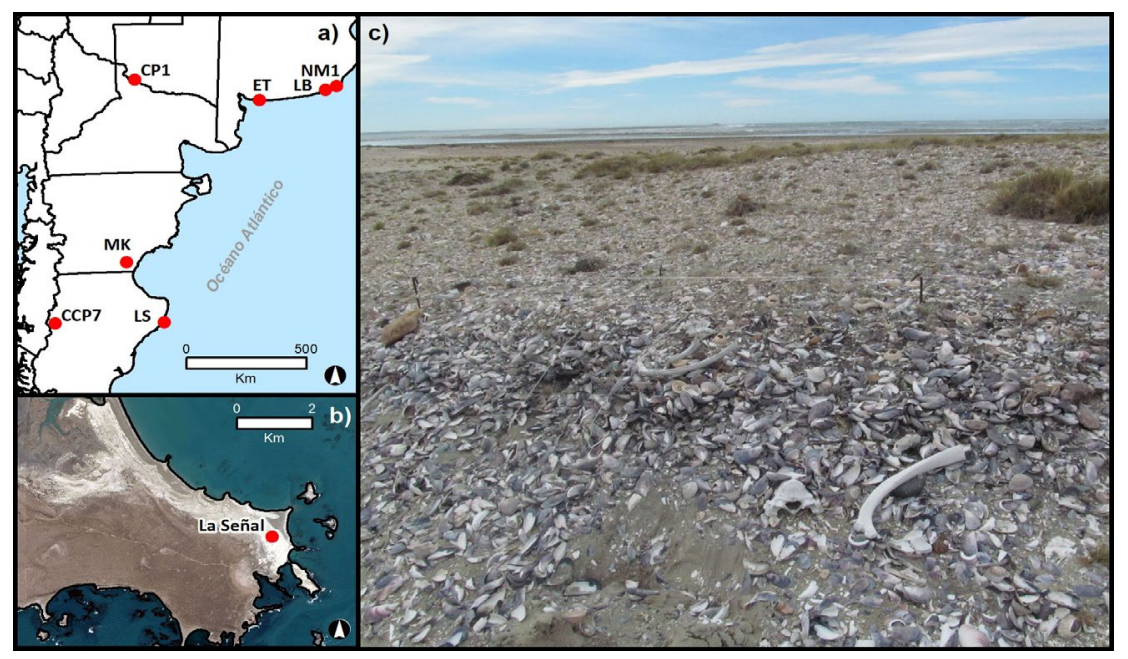

Figura 1. Sitio La Señal. Referencias: a) ubicación de los sitios mencionados en el texto, CCP7 = Cerro Casa de Piedra 7; LS = La Señal; $M K=$ colección Miksa-Knoop, ubicación relativa; $C P 1=$ Casa de Piedra 1; ET = El Tiburonero; LB = La Ballenera; y NM1 = Nutria Mansa 1; b) ubicación del sitio La Señal; y c) vista del sitio antes de la excavación del sondeo, en la parte superior se halló el diente fósil de tiburón.

Figure 1. La Señal archaeological site. References: a) location of the sites mentioned in the text, CCP7 = Cerro Casa de Piedra 7; $L S=L a$ Señal; $M K=$ Miksa-Knoop collection, relative location; $C P 1=$ Casa de Piedra 1; $E T=E$ I Tiburonero; $L B=L a$ Ballenera; and NM1 = Nutria Mansa 1; b) location of the La Señal site; and c) view of the site before excavation of the sounding, in the upper part, the fossil tooth of shark was found. 


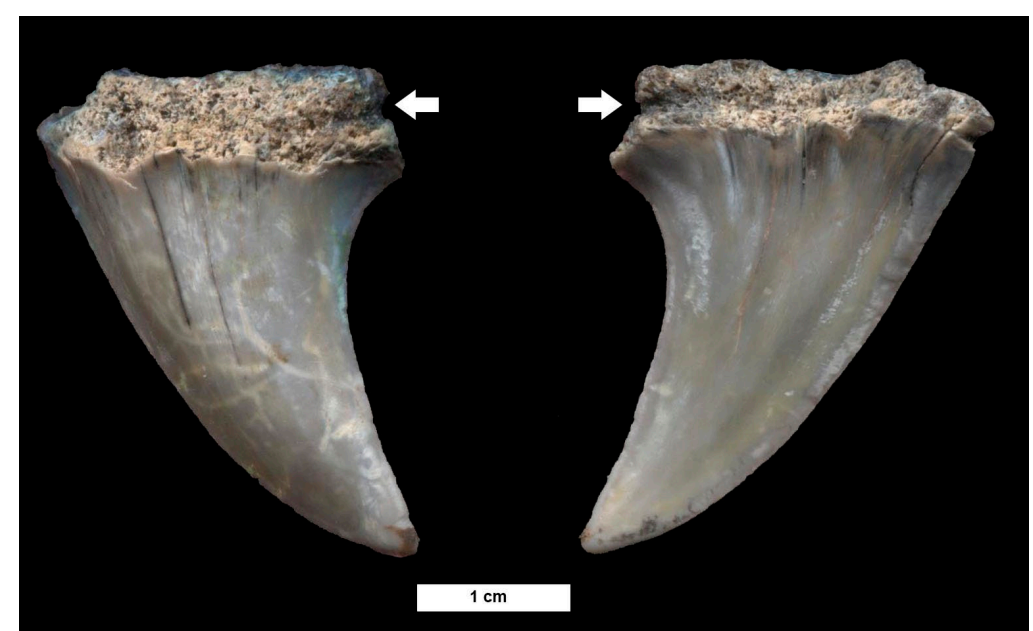

Figura 2. Vista lingual y labial del diente fósil de tiburón recuperado en el sitio La Señal. Las flechas blancas señalan las escotaduras en la raíz.

Figure 2. Lingual and labial view of the fossil tooth of shark recovered at La Señal archaeological site. White arrows point to the notches in the root.

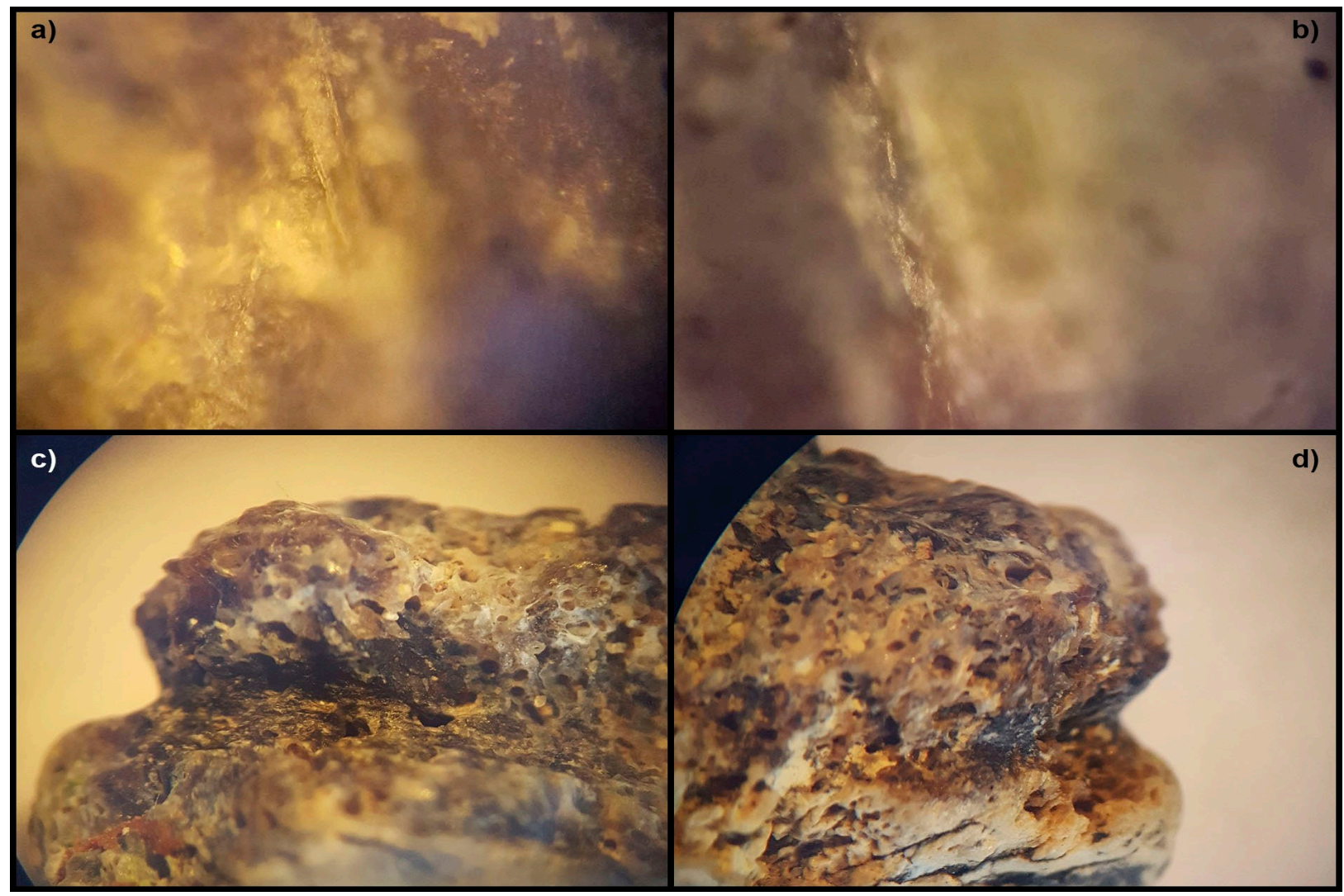

Figura 3. Vistas de los análisis microscópicos. Referencias: a) incisiones lineales multidireccionales; b) superficie pulida; c) detalle en vista lingual de la escotadura en el borde mesial; y d) detalle en vista labial de la escotadura en el borde mesial.

Figure 3. View of the microscopic analyses. References: a) multidirectional linear incisions; b) polished surface; c) detail in lingual view of the notch in the mesial border; and d) detail in labial view of the notch in the mesial border. 
afloramientos de la Formación Monte León son de 20 a $30 \mathrm{~km}$. Por otro lado, no existen referencias -de acuerdo a los pobladores locales- respecto del hallazgo de dientes fósiles en la zona de Punta Medanosa.

En cuanto al estudio del ejemplar identificado, cabe mencionar que se realizaron observaciones tanto a nivel macroscópico, usando lupa binocular, como a escala microscópica, mediante el empleo de un microscopio metalográfico marca Unión con aumentos de entre 100x y 300x. En función de estos análisis se comprobó la presencia de dos escotaduras simétricas ubicadas en los bordes mesial y distal de la pieza, en donde se juntan la corona y el hombro de la raíz del diente (Figura 2). Dichas escotaduras se podrían haber realizado mediante la utilización un instrumento lítico afilado, el cual habría dejado incisiones lineales multidireccionales, las cuales se observan a nivel microscópico (Figura 3.a). Algunos autores han planteado que muescas similares a las registradas se podrían producir de forma natural, aunque no han precisado de qué forma ni qué agentes intervendrían (Godfrey 2013). Asimismo, cabe mencionar que, en la cara lingual de la pieza, en la zona cercana al borde distal, se observa una incisión longitudinal a lo largo de la unión de la corona y la raíz (Figura 3.b). Esta incisión se registra también en la cara labial, aunque está más limitada a la escotadura del borde distal (Figura 3.c). Por último, en la escotadura del borde mesial se observaron también superficies redondeadas y pulidas concordantes con el contacto con sustancias blandas como el cuero, lo que podría indicar el uso de tientos de dicho material para confeccionar el engarce del diente (Figura 3.d). Como se mencionó antes, la raíz de la pieza se halla fracturada y buena parte de ella está ausente. Es probable que la misma se haya dañado siguiendo un posible debilitamiento producido por las incisiones realizadas para el engarce.

\section{Antecedentes}

Los antecedentes de registros de dientes de tiburón en contextos arqueológicos son muy escasos (Figura 1.a). En el sitio Cerro Casa de Piedra 7 (CCP7), en el centrooeste de la provincia de Santa Cruz se identificó un diente fósil del género Isurus en contextos asociados a ocupaciones de hace 9700 a 9000 años AP; el ejemplar presentaba daños estructurales en los bordes y el ápice, y estriaciones; dichos rasgos permitieron caracterizarlo como un instrumento (Castro et al. 2012). Por su parte, en la colección privada Miksa-Knoop, que cuenta con piezas recolectadas en el sureste de la provincia del Chubut, se reconoció un diente de tiburón fósil entero con corona y raíz de "Isurus" hastalis, la pieza presentaba dos incisiones en las puntas de la raíz que habrían permitido su uso como colgante y/o pendiente (Zubimendi et al. 2007). En la provincia de La Pampa, en el sitio Casa de Piedra 1 se recuperó un diente fósil sin evidencias de modificaciones antrópicas. Asimismo, en el sitio Nutria
Mansa 1 (provincia de Buenos Aires) se registraron dos dientes de tiburón blanco (Carcharodon carcharias) en un componente arqueológico datado en el Holoceno tardío (3100-2700 años AP) (Cione y Bonomo 2003; Bonomo 2006). Dichas piezas fueron modificadas mediante la realización de incisiones en las puntas de las raíces, las cuales se interpretaron como dispositivos empleados para el paso de hilos y así posibilitar el uso de los ejemplares como colgantes. Cabe mencionar que los bordes de uno de los dientes presentaban sus estrías erodadas, lo que también fue vinculado al uso del mismo como herramienta. En la misma provincia se ha identificado un diente fósil de Carcharodon carcharias en superficie, en inmediaciones del arroyo La Ballenera, en una línea de dunas litorales y otros ejemplares en el sitio superficial El Tiburonero, en médanos de Monte Hermoso (Cione y Bonomo 2003).

En el resto del mundo, el uso prehistórico de dientes de tiburón es más común respecto de los registros en Argentina. En Sudamérica, Centroamérica, Estados Unidos y el Pacífico se utilizaron dientes de tiburón fósiles y de especies actuales de diversas formas: con una o dos perforaciones en la raíz para usar como colgantes en collares; con escotaduras en los bordes para su enmangue y uso como puntas de proyectil; grandes cantidades de dientes alineados para manufacturar armas de mano características que se empleaban en conflictos altamente ritualizados; ejemplares sin modificaciones para el grabado y engarce de perlas; para la perforación de agujeros y/ o para la decoración de materiales más blandos (Betts et al. 2012; Colvin 2011; Drew et al. 2013; Lowery et al. 2011, entre otros).

\section{Consideraciones finales}

Los análisis realizados al diente fósil de "Isurus" hastalis que se registró asociado a un contexto arqueológico datado en ca. 2100 años AP en la localidad arqueológica Punta Medanosa, permiten afirmar que se trata de un objeto adorno-colgante. La materia prima provendría de alguno de los muchos afloramientos terciarios de la zona, los cuales presentan restos paleontológicos, por lo que su manufactura podría considerarse local. Como se comprobó, el fósil posee evidencias de modificaciones que generaron dos escotaduras en sus bordes y dos incisiones a lo largo de la raíz. Esto habría posibilitado la sujeción del diente mediante algún cordel o tiento y su consecuente uso como posible adorno corporal. Escotaduras similares a las registradas en el diente fósil identificado en el sitio La Señal, se han interpretado, en otras partes del mundo, como modificaciones realizadas en el marco de confección y utilización de las mismas como puntas de proyectil; sin embargo, en este caso, la morfología curvada del diente hace poco probable dicho uso. Como se mencionó antes, los antecedentes relacionados con el empleo de dientes fósiles de tiburón en el marco de ocupaciones y actividades de grupos humanos 
prehistóricos son muy escasos en la Argentina. Es por ello que todo nuevo hallazgo permite ampliar el estudio del uso de restos fósiles por parte de las poblaciones del pasado. En este sentido, cabe mencionar que dichos registros permiten visualizar con mayor amplitud el espectro de materias primas disponibles con las que los grupos humanos realizaron actividades tecnológicas, de consumo y/o de carácter simbólico (Bonomo 2006). A su vez, permite vislumbrar una mayor variabilidad respecto de las posibles utilidades y técnicas de formatización respecto de otros ejemplares analizados, los cuales presentan -como por ejemplo las piezas registradas en Nutria Mansa 1- incisiones completas con características diferenciales asociadas a las puntas de las raíces.

Finalmente, consideramos importante destacar que el diente fósil de tiburón con modificaciones antrópicas del sitio La Señal, constituye una pieza que será integrada a un estudio más amplio, el cual actualmente está en curso, sobre instrumentos confeccionados sobre huesos, valva y madera, los cuales se vinculan con aspectos sociales y/o simbólicos de las poblaciones patagónicas que habitaron la costa norte de Santa Cruz en tiempos prehistóricos. En tal sentido, el diente de tiburón amplía la variabilidad de materias primas y tipos de objetos simbólicos -en este caso en particular, adorno-colgantes- existentes en el área de estudio.

La Plata 28 de febrero de 2019

\section{Agradecimientos}

Agradecemos a la familia Vidal, dueños de la estancia El Amanecer, al Museo Municipal Mario Brozoski y la Municipalidad de Puerto Deseado, al Consejo Agrario de Santa Cruz, delegación Puerto Deseado por las ayudadas brindadas durante los trabajos de campo. También agradecemos a Alberto Cione y Sergio Bogan por sus sugerencias y ayuda en la identificación del diente. Por último, estamos agradecidos a los dos evaluadores anónimos, cuyos comentarios permitieron mejorar el manuscrito. Los estudios se realizaron dentro de los proyectos "Estudios regionales en el eje Deseado para definir los rangos de acción de grupos cazadores recolectores costeros en el marco de la ocupación humana del Holoceno de Patagonia (N739)"; PICT 2014-3591 "Localidad arqueológica Punta Medanosa: cambios geomorfológicos y arqueológicos a lo largo del Holoceno medio y tardío" y PICT 2016-0404 "Arqueología de la Cuenca inferior del río Deseado: La ocupación de abrigos rocosos en el marco de la explotación de recursos litorales".

\section{Bibliografía}

Arratia, G., A. L. Cione (1996). The fish fossil record of Southern South America. Münchener Geowissenschaft Abhanlungen, 30: 9-72
Betts, M., S. Blair y D. Black (2012). Perspectivism, mortuary symbolism, and human-shark relationships on the Maritime Peninsula. American Antiquity, 77(4): 621-645.

Bonomo, M. (2006). Un acercamiento a la dimensión simbólica de la cultura material en la región pampeana. Relaciones de la Sociedad Argentina de Antropología $\mathrm{XXXI}: 89-115$

Castro, A., A. L. Cione, T. Civalero y M. De Nigris (2012). A Fossil Shark Tooth in Early Contexts of Cerro Casa de Piedra 7, Southwest Patagonia Argentina. L. Miotti, M. Salemme, N. Flegenheimer, y T. Goebel (Eds.), Southbound Late Pleistocene Peopling of Latin America: 165-176. Center for the Study of the First Americans, Texas A\&M University, College Station.

Cione, A., M. Bonomo. (2003). Great white shark teeth used as pendants and possible tools by Early-Middle Holocene terrestrial mammal hunter-gatherers in the eastern Pampas (Southern South America). International Journal of Osteoarchaeology, 13: 222-231.

Cione, A. L., D. Cabrera y M. Barla. (2012). Oldest record of the Great White Shark (Lamnidae, Carcharodon; Miocene) in the Southern Atlantic. Geobios, 45(2): 167172.

Colvin, G. (2011). The presence, source and use of fossil shark teeth from Ohio archaeological sites. Ohio Archaeologist, 61(4):26-46.

Constante, M. (2001). "Geomorfología y geología de Ensenada Ferrer, Provincia de Santa Cruz". Departamento de Ciencias Geológicas, Universidad de Buenos Aires, tesis de Licenciatura.

Drew, J., C. Philipp y M. Westneat (2013). Shark tooth weapons from the 19th century reflect shifting baselines in Central Pacific predator assemblies. PlosOne, 8(4): e59855.

Ehret, D., B. Macfadden, D. Jones, T. DeVries, D. Foster y R. Salas-Gismondi (2012). Origin of the white shark Carcharodon (Lamniformes: Lamnidae) based on recalibration of the Neogene Pisco Formation of Peru. Palaeontology, 55: 1139-1153.

Fujita, H., C. Cáceres-Martínez y A. F. Ainis (2017). Pearl Ornaments from the Covacha Babisuri Site, Espíritu Santo Island, Baja California, Mexico. Pacific Coast Archaeological Society Quarterly, 53(2-3): 63-86.

Godfrey, S. (2013). Modified fossil Mako teeth. The Ecphora, 28(4): 2-4.

Lowery, D., S. Godfrey y R. Eshelman. (2011). Integrated 
geology, paleontology, and archaeology: native american use of fossil shark teeth in the Chesapeake bay. Archaeology of Eastern North America, 39: 93-108.

Medina, R., M. Aguirre, J. Codignotto, S. Richiano y L. Mormeneo (2014). Geoformas, malacofauna y evolución costera durante el Holoceno en Ensenada Ferrer (Santa Cruz, Patagonia, Argentina). Revista de la Asociación Geológica Argentina 71(1): 69-81.

Panza, J., M. Márquez y M. Godeas (1994). Descripción de la Hoja Geológica 4966-I y II "Bahía Laura", Provincia de Santa Cruz. Servicio Geológico Minero Argentino, Instituto de Geología y Recursos Minerales, Buenos Aires.

Ward, D. J., C. G. Bonavia (2001). Additions to, and a review of, the Miocene shark and ray fauna of Malta. The Central Mediterranean Naturalist, 3: 131-146.

Zubimendi, M. A., P. Ambrústolo y M. A. Andolfo (2007). Informe de las actividades de clasificación y catalogación de la colección privada Miksa-Knoop. E. Knoop (ed.), Arte lítico Tehuelche. Legado de la Patagonia: 77-187, Editorial Dunken, Buenos Aires. 